\section{A case report of acute myeloid} leukemia and neurofibromatosis 1

\author{
Chiara Sartor, Cristina Papayannidis, \\ Maria Chiara Abbenante, Antonio Curti, \\ Nicola Polverelli, Emanuela Ottaviani, \\ Ilaria lacobucci, Viviana Guadagnuolo, \\ Giovanni Martinelli \\ Department of Experimental, Diagnostic \\ and Specialty Medicine, University of \\ Bologna, Italy
}

\begin{abstract}
We report a case of a 65 -year old patient affected by neurofibromatosis 1 , documented by the presence of germ-line mutation on the $N F 1$ gene, who developed various hyperproliferative malignant and benign diseases. He was brought to our attention for the diagnosis of acute myeloid leukemia revealed by major fatigue and dyspnea. The disease characteristics at diagnosis were hyperleukocytosis and complex karyotype with the inversion of the chromosome 16 , classifying as a high-risk leukemia. The association between leukemia and neurofibromatosis 1 is controversial and needs to be further investigated. Nevertheless, such patients present a wide number of comorbidities that make therapeutic strategies most difficult.
\end{abstract}

\section{Introduction}

\section{Neurofibrosis type 1}

Neurofibromatosis type 1 (NF1), otherwise known as Von Recklinghausen disease, is a neurocutaneous genetic disorder with an autosomal dominant transmission. In $50 \%$ of cases, the mutation of the NF1 gene arises de novo. ${ }^{1}$ The prevalence of NF1 has been estimated approximately as one in 3500 , the penetrance is $100 \%$ associated with a high phenotypic variability. The most common clinical features are café au lait macules, skinfold freckling, Lisch nodules (hamartomas of the iris) and neurofibromas, but, being a multisystem disorder, any organ system can be affected. ${ }^{2}$ The association between NF1 and various pathologies has been established, such as orthopedic abnormalities, neurological and psychiatric disorders, congenital cardiac defects and vasculopathy, ophthalmological diseases and an increased cancer risk. ${ }^{3} \mathrm{~A}$ prospective study has evaluated such predisposition as a 2.7 -fold increased overall risk of cancer malignancies in patients with NF1, and a cumulative risk of $20 \%$ by the age of 50 years. ${ }^{4}$ The most represented tumors derive from tissues of neuroec- todermal origin such as central nervous system, peripheral nerve sheath tumors and pheochromocytoma. Malignancies such as juvenile monocytic leukemia, gastrointestinal stromal tumors, rhabdomyosarcoma have also been strongly associated to NF1, while the onset of breast cancer, melanoma, acute lymphoblastic leukemia, non-Hodgkin's lymphoma, carcinoid and Wilm's tumors is considered a possible association, currently under evaluation. $^{2}$

\section{Acute myeloid leukemia}

AML is a rare aggressive neoplasm of the hematopoietic myeloid stem cell. The incidence of AML development increases with age: less than $4 \%$ in patients under 60 years of age and becomes over $19 \%$ in patients over 75 years of age. The common clinical presentation is related to the invasion of the bone marrow by blast cells, which blocks the normal hematopoietic process, leading to anemia (shortage of erythrocytes), leukopenia and thrombocytopenia. Resultant symptoms and signs include fatigue, shortness of breath, infections and bleeding.

\section{Case Report}

A 68-year old man was admitted to our hematology department with the diagnosis of AML revealed by routine laboratory tests performed in presence of a major fatigue and a dyspnea at minimum effort. The results showed a hemoglobin level of $7.7 \mathrm{~g} / \mathrm{dL}$, a total leukocyte count of $14 \times 10 \%$ with $50 \%$ blast cells, and a platelet count of $114 \times 10^{9} / \mathrm{L}$. The bone marrow biopsy diagnosed acute myeloid leukemia. The morphology and immunophenotyping established the expression of markers connected with precursor stage and monocytic differentiation (FAB M2/M4).

The anamnesis of the patient was most complex, beginning with the diagnosis of neurofibromatosis type 1 at the age of 30 years for the presence of multiple neurofibromas and tache au lait macules covering the whole body. The mutation had most likely arisen de novo as there is no knowledge of close relatives presenting any of the classical cutaneous characteristics. The physical examination confirmed the presence of multiple neurofibromas, tache au lait macules and skinfold freckling. No liver or spleen enlargement was found. The clinical anamnesis reported various hyperproliferative disorders involving different organs. The patient has a history of hypertension since young age, which has led to the first heart attack at the age of 41 years. Following episodes at the age of 58 years, associated with a possible genetic predisposition for heart defects due to NF1, a dilated cardiomyopa-
Correspondence: Giovanni Martinelli, Department of Experimental, Diagnostic and Specialty Medicine, University of Bologna, via Massarenti 9, 40138 Bologna, Italy.

Tel. +39.051 .6363829 - Fax: +39.051 .6364037$

E-mail: giovanni.martinelli2@unibo.it

Key words: acute myeloid leukemia, neurofibromatosis type 1, NF1, Von Recklinghausen disease.

Conflict of interests: the authors declare no potential conflict of interests.

Received for publication: 2 April 2013.

Accepted for publication: 16 April 2013.

This work is licensed under a Creative Commons Attribution NonCommercial 3.0 License (CC BYNC 3.0).

(c) Copyright C. Sartor et al., 2013

Licensee PAGEPress, Italy

Hematology Reports 2013; 5:e8

doi:10.4081/hr.2013.e8

thy was revealed for which he received a heart transplant at the age of 59 years. He has been in treatment with cyclosporine since then. A multiple series of benign and malign tumors followed. The patient has undergone: i) a right hemithyroidectomy for an adenomatous thyroid hyperplasia; ii) a right hemicolectomy for an intestinal polyp classified as WHO low-grade dysplasia; iii) the excision of a melanoma Clark level II localized on the back; and iv) an inferior left lung lobectomy associated with lymphadenectomy for a bronchioloalveolar non-mucinous carcinoma. The patient also presented hypertrophy of the prostate (athough this is most common in men his age) and a slight kidney failure. The last hyperproliferative disorder diagnosed was the acute myeloid leukemia for which he was admitted to the hematology department. On entering our unit, the patient presented $9.0 \times 10^{9} / \mathrm{L}$ of hemoglobin, $55 \times 10^{9} / \mathrm{L}$ platelets and $21.6 \times 10^{9} / \mathrm{L}$ leucocytes ( $1 \%$ neutrophils and $75 \%$ blast cells). The cytogenetic analysis showed a complex karyotype, with the inversion of the chromosome 16, and the molecular biology evaluation revealed the rearrangement of $C B F B$ MYH11. NMP1 and FLT3 genes were analyzed and these resulted wild-type. Considering the age and important comorbidities presented, the decision was taken to begin induction treatment with intermediate-dose cytarabine (Ara-C). The complete morphological remission was obtained after the first cycle and maintained during the second and third, without major complications during cytopenia. The patient is currently beginning the fourth cycle of therapy and is in good clinical condition. 


\section{Discussion}

NF1 is a tumor suppressor gene localized on 17q11.2. It encodes neurofibromin, a negative regulator of proto-oncogene Ras. The loss of neurofibromin promotes Ras activity leading to constitutive downstream signaling and increased uncontrolled cell growth. ${ }^{5}$ Hyperproliferation is a mechanism that involves every organ system leading to the predisposition for both cancerous and non-cancerous disorders. It is at the base of the so called Ras-opathies, a group of inherited disorders that share a germ-line mutation of the RAS-MAPK pathway, to which NF1 belongs. ${ }^{6}$

The association between hematologic malignancies and germ-line mutations of NF1 has been established in the pediatric setting. Children with neurofibromatosis 1 have a 500fold increased risk of developing a rare form of leukemia, known as juvenile myelomonocytic leukemia (JMML); a higher incidence of nonHodgkin's lymphoma and acute lymphoblastic leukemia has also been reported., 3,8

As far as adult patients are concerned, even though the risk of malignancies in patients affected by NF1 is well known and increases with age, most cancer cases reported are not hematologic neoplasms. ${ }^{4}$ Nevertheless the association between both leukemia and lymphoma has been described., ${ }^{4,-12}$ Of these, few cases concerned acute myeloid leukemia, although the association could be supported by a wide number of studies on somatic mutations of the NF1 gene and AML. ${ }^{13-16}$ In subjects with NF1 mutations, the loss of the remaining $N F 1$ allele is a frequent event in AML, as it is a gene subject to copy number alteration. ${ }^{17}$ The frequency of NF1 null AML is estimated to be $7 \% .^{13}$ Given the incidence of neurofibromatosis type 1 in the population, and that of AML, more studies are needed to establish a direct connection between the two. In our case, the myeloid lineage and the presence of myelomonocytic precursors resembled the morphology of JMML, supporting the hypothesis of a connection between the patient's leukemia and NF1.

\section{Conclusions}

What does seems to be certain is that with a patient presenting NF1, much attention has to be given to the anamnesis and the overall global clinical evaluation. NF1 is a common inherited syndrome with a high number of potentially associated pathologies and malignancies. ${ }^{3}$ Our patient reported important vasculopathy, hypertension and heart defects, widely described in literature as a consequence of NF1. ${ }^{18-20}$ Moreover, he had undergone important surgery on more than one occasion for antecedent neoplasms. These patients are, therefore, extremely fragile and any therapy proposed needs to be evaluated and managed according to the elevated number of comorbidities present.

\section{References}

1. Littler M, Morton NE. Segregation analysis of peripheral neurofibromatosis (NF1). J Med Genet 1990;27:307-10.

2. Boyd KP, Korf BR, Theos A. Neurofibromatosis type 1. J Am Acad Dermatol 2009; 61:1-14.

3. Yohay K. Neurofibromatosis type 1 and associated malignancies. Curr Neurol Neurosci Rep 2009;9:247-53.

4. Walker L, Thompson D, Easton D, et al. A prospective study of neurofibromatosis type 1 cancer incidence in the UK. Br J Cancer 2006;95:233-8.

5. Williams VC, Lucas J, Babcock MA, et al. Neurofibromatosis type 1 revisited. Pediatrics 2009;123:124-33.

6. Tartaglia M, Gelb BD. Disorders of dysregulated signal traffic through the RASMAPK pathway: phenotypic spectrum and molecular mechanisms. Ann NY Acad Sci 2010;1214:99-121.

7. Stiller CA, Chessells JM, Fitchett M. Neurofibromatosis and childhood leukaemia/lymphoma: a population-based UKCCSG study. Br J Cancer 1994;70:96972.

8. de Vries AC, Zwaan CM, van den Heuvel-
Eibrink MM. Molecular basis of juvenile myelomonocytic leukemia. Haematologica 2010;95:179-82.

9. Goerg C, Goerg K, Pflueger KH, Havemann K. Neurofibromatosis and acute monocytic leukemia in adults. Cancer 1989;64:17179.

10. Van Baren N, Issa A, Delannoy A. Von Recklinghausen neurofibromatosis and hematologic malignancies: 2 case reports in adulthood. Acta Clin Belg 1993;48:16470.

11. Julia A, Ayguasanosa J, Blanco A. Familial neurofibromatosis type I and adult acute lymphocytic leukemia. Haematologica 1999;84:472-3.

12. Lueangarun S, Auewarakul CU. Diffuse large B cell lymphoma presenting as Horner's syndrome in a patient diagnosed with neurofibromatosis type 1: a case report and review of the literature. $\mathrm{J}$ Med Case Rep 2012;6:8.

13. Parkin B, Ouillette P, Wang Y, et al. NF1 inactivation in adult acute myelogenous leukemia. Clin Cancer Res 2010;16:413547.

14. Mullally A, Ebert BL. NF1 inactivation revs up Ras in adult acute myelogenous leukemia. Clin Cancer Res 2010;16:4074-6.

15. Johannessen CM, Reczek EE, James MF, et al. The NF1 tumor suppressor critically regulates TSC2 and mTOR. Proc Natl Acad Sci USA 2005;102:8573-8

16. Mrozek K. Cytogenetic, molecular genetic, and clinical characteristics of acute myeloid leukemia with a complex karyotype. Semin Oncol 2008;35:365-77.

17. Walter MJ, Payton JE, Ries RE, et al. Acquired copy number alterations in adult acute myeloid leukemia genomes. Proc Natl Acad Sci USA 2009;106:12950-5.

18. $\mathrm{Xu}$ J, Ismat FA, Wang $\mathrm{T}$, et al. Cardiomyocyte-specific loss of neurofibromin promotes cardiac hypertrophy and dysfunction. Circ Res 2009;105:304-11.

19. Gitler AD, Epstein JA. Regulating heart development: the role of Nf1. Cell Cycle 2003;2:96-8.

20. $\mathrm{Xu}$ J, Ismat FA, Wang T, et al. NF1 regulates a Ras-dependent vascular smooth muscle proliferative injury response. Circulation 2007;116:2148-56. 